\title{
Treatment effectiveness of pasireotide on health-related quality of life in patients with Cushing's disease
}

\author{
Susan M Webb, John E Ware ${ }^{1,2}$, Anna Forsythe ${ }^{3}$, Min Yang ${ }^{4}$, Xavier Badia ${ }^{5}$, \\ Lauren M Nelson ${ }^{6}$, James E Signorovitch ${ }^{4}$, Lori McLeod ${ }^{6}$, Mario Maldonado7, \\ Wojciech Zgliczynski ${ }^{8}$, Christophe de Block ${ }^{9}$, Lesly Portocarrero-Ortiz ${ }^{10}$ and \\ Monica Gadelha ${ }^{11}$
}

Department of Medicine/Endocrinology, Centre for Biomedical Research on Rare Diseases (CIBERER Unit 747), Hospital Sant Pau, Universitat Autònoma de Barcelona, Barcelona, Spain, 'Department of Quantitative Health Sciences, University of Massachusetts Medical School, Worcester, Massachusetts, USA, ${ }^{2}$ John Ware Research Group, Inc., Worcester, Massachusetts, USA, ${ }^{3}$ Novartis Pharmaceuticals Corporation, East Hanover, New Jersey, USA, ${ }^{4}$ Analysis Group, Inc., Boston, Massachusetts, USA, ${ }^{5}$ IMS Health, Barcelona, Spain, ${ }^{6}$ RTI Health Solutions, Research Triangle Park, North Carolina, USA, ${ }^{7}$ Novartis Pharma AG, Basel, Switzerland, ${ }^{8}$ Department of Endocrinology, Centre for Postgraduate Medical Education, Warsaw, Poland, ${ }^{9}$ Department of Endocrinology, Diabetology and Metabolism, University Hospital, Antwerp, Belgium, ${ }^{10}$ National Institute of Neurology and Neurosurgery 'Manuel Velasco Suárez', Mexico City, Mexico and ${ }^{11}$ Division of Endocrinology, Medical School, Federal University of Rio de Janeiro, Rio de Janeiro, Brazil

\author{
Correspondence \\ should be addressed \\ to M Yang \\ Email \\ myang@analysisgroup.com
}

\begin{abstract}
Objective: Cushing's disease (CD) can significantly impair patients' health-related quality of life (HRQOL). This study investigated the treatment effectiveness of pasireotide on HRQOL of CD patients, and assessed the relationships between HRQOL and urinary free cortisol (UFC) and CD-related signs and symptoms.

Design: In this phase III, randomized, double-blind study, patients with UFC $\geq 1.5 \times$ upper limit of normal (ULN) received s.c. pasireotide 600 or $900 \mu \mathrm{g}$ twice daily. The trial primary endpoint was UFC at or below ULN at month 6 without dose titration. Open-label treatment continued through month 12. HRQOL was measured using the Cushing's Quality of Life Questionnaire (CushingQoL) instrument at baseline and follow-up visits until month 12 during which clinical signs and features of CD, and the Beck Depression Inventory II (BDI-II), were also collected.

Methods: Pearson's/Spearman's correlations between changes in CushingQoL and changes in clinical signs and symptoms were assessed. Changes in CushingQoL and the proportion of patients achieving a clinically meaningful improvement in CushingQoL were also compared among patients stratified by mean UFC (mUFC) control status (controlled, partially controlled, and uncontrolled) at month 6 . Analyses were also conducted at month 12 , with multivariable adjustment for baseline characteristics and CushingQoL.

Results: Change in CushingQoL was significantly correlated with changes in mUFC ( $r=-0.40), \mathrm{BMI}(r=-0.39)$, weight $(r=-0.41)$, and BDI-II $(r=-0.54)$ at month 12 but not at month 6 . The percentage of CushingQoL responders at month 12 based on month 6 mUFC control status were as follows: $63,58.8$, and $37.9 \%$ in the controlled, partially controlled, and uncontrolled groups respectively. Adjusted CushingQoL scores at month 12 were 58.3 for controlled patients ( $\Delta=11.5$ vs uncontrolled, $P=0.012$ ) and 54.5 for partially controlled patients ( $\Delta=7.7$ vs uncontrolled, $P=0.170$ ). Conclusions: Pasireotide treatment can result in a meaningful HRQOL improvement among those who complete a 12-month treatment period, most often among patients achieving biochemical control.




\section{Introduction}

Cushing's disease (CD) has a significant impact on the physical, cognitive, emotional, and social well-being of patients $(1,2,3,4,5,6)$. Although rare, CD is an extremely debilitating neuroendocrine condition caused by an adrenocorticotropic hormone (ACTH)-secreting pituitary adenoma. The surplus ACTH secreted by the tumor stimulates the adrenal glands to produce excess cortisol. The hypercortisolism and its complications lead to a wide range of signs and symptoms, including central obesity, facial fullness, facial rubor, hirsutism, striae, development of a posterior cervical fat pad ('buffalo hump'), delayed wound healing and bruising, generalized weakness and fatigue, wasting of proximal muscles, menstrual disorders, hypertension, glucose intolerance, diabetes mellitus, osteoporosis, gonadal dysfunction, sleep disturbances, depression, and cognitive dysfunction (7). Previous studies report that the negative impact of CD on health-related quality of life (HRQOL) can persist despite successful endocrine treatment $(2,8)$. Therefore, in addition to assessing biochemical response to a treatment, such as urinary free cortisol (UFC) levels, it is important to understand the severity and impact of CD on patients in their daily lives and to evaluate the impact of the treatment on HRQOL.

Transsphenoidal surgery is the primary treatment for CD (7). However, not all patients with CD are candidates for surgery, and surgery is not always successful; $25-30 \%$ of surgical patients fail to achieve long-term remission (9). Pasireotide (SOM230), a somatostatin analog that targets somatostatin receptors $1,2,3$, and 5 , is the first-approved drug therapy for CD that targets the pituitary adenoma. Activation of somatostatin receptors has been shown to inhibit ACTH secretion in vitro. In a phase II study, s.c. injections of pasireotide in CD patients demonstrated reduction in UFC after 15 days of treatment (10). Based on these findings, a phase III randomized, multicenter, double-blind clinical trial was conducted to investigate the safety and efficacy of pasireotide in patients with CD (1).

The objectives of this study were to investigate the treatment effectiveness of pasireotide on HRQOL, and to assess the relationship between HRQOL and UFC control and other indicators of CD severity using data from the phase III clinical trial of patients with CD.

\section{Subjects and methods}

\section{Study sample}

The phase III clinical trial enrolled 162 adult patients (aged $\geq 18$ years) with confirmed persistent/recurrent or de novo CD. Patients were required to have a 24-h mean UFC (mUFC) at least $1.5 \times$ upper limit of normal (ULN; $145 \mathrm{nmol} / 24 \mathrm{~h}$ ), morning plasma ACTH at least $5 \mathrm{ng} / \mathrm{l}$, and a confirmed pituitary source of the endogenous hypercortisolism. Patients were randomized to twice daily (b.i.d.) s.c. pasireotide $600 \mu \mathrm{g}(n=82)$ or $900 \mu \mathrm{g}(n=80)$ after a 30-day screening period and appropriate washout of cortisol-lowering medications. Patients continued their randomly assigned dose until month 6 (double-blind treatment phase) if their month 3 mUFC was $\leq 2 \times$ ULN and less than or equal to their baseline mUFC. Patients not meeting mUFC criteria at month 3 were unblinded and their dose was increased by an additional $300 \mu \mathrm{g}$ b.i.d. At month 6, all patients entered an open-label phase that continued up to month 12 . During this open-label phase, the dose could be increased by $300 \mu \mathrm{g}$ b.i.d. (to a maximum of $1200 \mu \mathrm{g}$ b.i.d.) at any time if UFC was above the ULN. Dose reductions of $300 \mu \mathrm{g}$ b.i.d. for drug-related adverse effects were permitted throughout the study. The primary trial endpoint was UFC at or below ULN at month 6 without dose up-titration. Per protocol, patients who had dose up-titration before month 6 were automatically assigned the mUFC status 'uncontrolled' at month 6. There were 78 patients who completed the 12-month trial (Fig. 1). Missing data were not imputed for the analyses in this study.

The trial was approved by the Institutional Review Board at each participating center and was conducted in accordance with the International Conference on Harmonisation Guidelines for Good Clinical Practice and the Declaration of Helsinki. All patients provided written informed consent (ClinicalTrials.gov: NCT00434148). Details of the trial design and primary outcomes have been reported elsewhere (1).

\section{Measures}

Demographic information, including age, sex, and ethnicity, were collected at baseline. UFC levels were measured at baseline, monthly up to month 6 and at months 9 and 12. Patients completed the Cushing's Quality of Life Questionnaire (CushingQoL), CD-related clinical signs and symptoms assessment, and the Beck Depression Inventory II (BDI-II) at baseline and months 3,6 , and 12 .

CushingQoL - CushingQoL is a patient-reported HRQOL instrument that was specifically developed for 


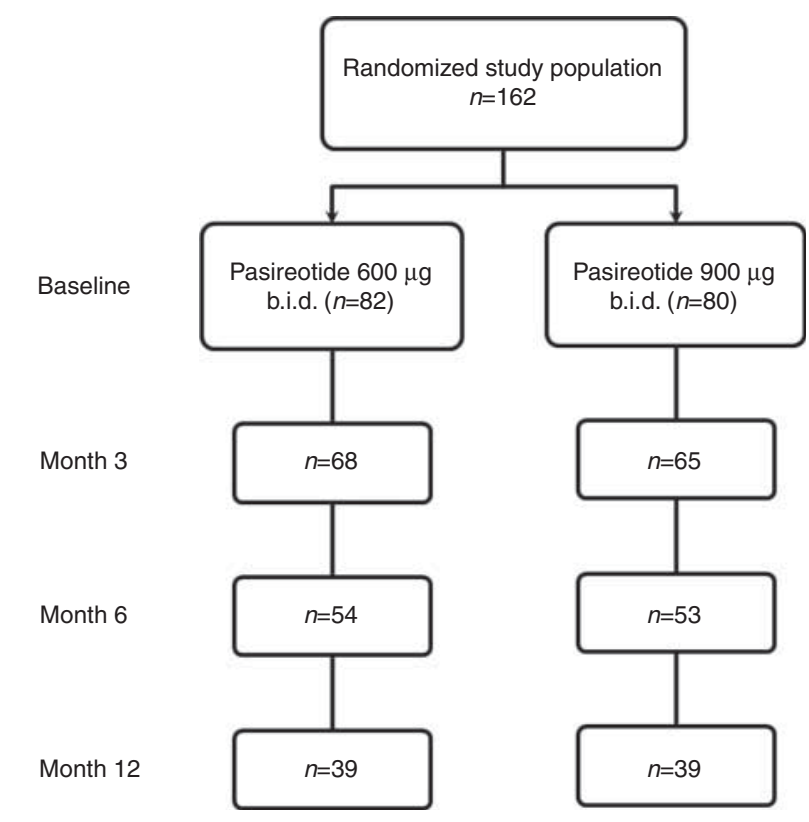

\section{Figure 1}

Patient flow chart. In the first 3 months, 29 patients discontinued the study: 13 because of adverse events, four because of lack of efficacy, nine because of withdrawal of consent, and three because of a protocol violation. By month 6 , an additional 26 patients had discontinued the study: seven because of adverse events, 15 because of lack of efficacy, and four because of withdrawal of consent. By month 12, an additional 29 patients had discontinued the study: six because of adverse events, 18 because of lack of efficacy, four because of withdrawal of consent, and one because of a protocol violation.

and validated in patients with $\mathrm{CD}(6,11)$. The 12 -item, patient-completed instrument has a 4 -week recall period and includes items on sleep difficulties, pain, delayed wound healing, bruising, irritability, insecurity, concern about physical appearance, difficulty in maintaining social engagements and daily activities, memory, and concern about future health. Item scores, scaled from 1 to 5 , are summed and standardized to form the CushingQoL score ranging from 0 to 100 . Higher CushingQoL scores indicate more favorable HRQOL. An improvement in CushingQoL score $>10.1$ has been estimated as an initial threshold for a clinically meaningful change based on a distributionbased method (12) for defining a minimally important difference (MID) (13).

Mean UFC $>$ The mUFC was calculated using four $24 \mathrm{~h}$ urine samples collected during a 2-week time period before baseline visit and visits at months 3,6 , and 12 . For this study, clinical response status was determined based on the mUFC level as follows:

i) UFC controlled: mUFC $\leq$ ULN

ii) partially controlled: mUFC $>$ ULN and $\geq 50 \%$ reduction from baseline

iii) uncontrolled: mUFC $>$ ULN and $<50 \%$ reduction from baseline.

In addition, the following definitions were used to categorize UFC severity:

i) normal: mUFC from lower limit of normal to $\leq$ ULN

ii) mild hypercortisolism: mUFC $>$ ULN to $<2 \times$ ULN

iii) moderate hypercortisolism: $\mathrm{mUFC} \geq 2 \times \mathrm{ULN}$ to $<5 \times$ ULN

iv) severe hypercortisolism: $\mathrm{mUFC} \geq 5 \times$ ULN to $<10 \times$ ULN

v) very severe hypercortisolism: $\mathrm{mUFC} \geq 10 \times \mathrm{ULN}$.

Clinical signs and features $>$ Patients' BMI $\left(\mathrm{kg} / \mathrm{m}^{2}\right)$, weight $(\mathrm{kg})$, and waist circumference $(\mathrm{cm})$ were assessed. Sitting and standing systolic and diastolic blood pressure (mmHg), HbA1c, and fasting plasma glucose (FPG) were also collected. Clinical features associated with hypercortisolism were assessed through clinician-rated photographs taken at all visits. At each site, a physician with expertise in Cushing's syndrome (CS), blinded to the treatment allocation and visit schedule, reviewed the photographs and evaluated physical characteristics, including facial rubor, striae, bruising, supraclavicular fat pad, and dorsal fat pad, using the scale none, 0 ; mild, 1 ; moderate, 2 ; or severe, 3 .

Beck depression inventory II $\boldsymbol{\sim}$ The patient-reported BDI-II (14) measures the intensity of depression over the past 2 weeks. The BDI-II has 21 items, each corresponding to a symptom of depression and rated from 0 to 3 . Responses are summed to yield a total score ranging from 0 to 63 (with higher scores indicating more severe depression). Consistent with previous literature, patients were categorized into four groups based on BDI-II score (14). These included minimal depression (0-13), mild depression (14-19), moderate depression (20-28), and severe depression (29-63).

\section{Analysis}

The core analysis used data from the 78 patients who completed the 12-month trial. Baseline demographic and clinical characteristics were reported overall and 
by treatment arm. Analyses of the relationship between pasireotide treatment effectiveness and HRQOL were conducted using the pooled sample from both treatment arms.

To evaluate the relationship between changes in CushingQoL and mUFC over time, changes in CushingQoL were estimated alongside with the changes in mUFC at baseline, months 3, 6, and 12 among completers and among all patients with measurements at those time points as a sensitivity analysis. Mean changes in CushingQoL by hypercortisolism level were also assessed at month 6 , the primary endpoint with a double-blind study design, and month 12, the end of the trial. Correlations were computed, with correlation coefficients of 0.10 , $0.10-0.5$, and $>0.5$ representing weak, moderate, and strong associations respectively (15). Pearson product moment correlation coefficients or Spearman's ranked correlation coefficients when the distributions were nonnormal were computed.

Paired $t$-tests were performed to evaluate the change in CushingQoL. An effect size was computed using Cohen's $d$ (15) to describe the strength of change in CushingQoL at months 6 and 12. Effect sizes of $0.2,0.5$, and 0.8 represented small, medium, and large degrees of change respectively (15). In addition, paired $t$-tests were conducted to assess the improvement observed on each of the 12 CushingQoL items from baseline to month 12 . Multivariable regression analysis was conducted to assess the factors (i.e. age, gender, baseline CushingQoL score, and hyperglycemia status - defined by HbA1c $\geq 6.5 \%$ or FPG $\geq 126 \mathrm{mg} / \mathrm{dl}$ - at month 12) associated with the CushingQoL score at month 12 and to assess adjusted associations between CushingQoL scores and mUFC control status. A sensitivity analysis was conducted by including the dose of pasireotide (600 vs $900 \mu \mathrm{g}$ ) as one of the control variables.

Patients were classified as CushingQoL responders if they experienced an improvement from baseline in CushingQoL scores $\geq 10.1$ MID threshold (13). The percentage of patients classified as CushingQoL responders by month 12 were compared among subgroups by UFC control status at months 6 and 12. Finally, CushingQoL score changes from baseline to month 12 were compared with MID in patients who were controlled, partially controlled, and uncontrolled at 6 and 12 months.

To evaluate the association between changes in CushingQoL scores and other clinical signs, features, and depression, correlations across treatment arms were computed with changes in BMI, weight, waist circumference, standing and sitting systolic and diastolic blood pressure, facial rubor, striae, bruising, supraclavicular fat pad, dorsal fat pad, and BDI-II scores.

\section{Results}

\section{Patients' characteristics}

Baseline characteristics of the study completers are presented in Table 1. Among the 78 study completers, patients were primarily female $(86 \%)$ and white $(82 \%)$, with an average age of 40.7 years and a persistent/ recurring CD status (85\%). Baseline mUFC ranged from 195.0 to $5010.3 \mathrm{nmol} / 24 \mathrm{~h}$ (mean \pm s.D. $=782.0 \pm 873.6$, median $=510$ ). In this sample, $19 \%$ of patients had mild hypercortisolism, 47\% had moderate hypercortisolism, $23 \%$ had severe hypercortisolism, and 11\% had very severe hypercortisolism.

The average systolic blood pressure was $130 \mathrm{mmHg}$ and the average diastolic blood pressure was $86 \mathrm{mmHg}$,

Table 1 Baseline characteristics of the study sample.

\begin{tabular}{|c|c|}
\hline Characteristic & $\begin{array}{c}\text { Study sample } \\
\quad(n=78)\end{array}$ \\
\hline Age (years), mean (s.D.) & $40.7(10.7)$ \\
\hline Female, $n(\%)$ & $67(85.9)$ \\
\hline White, $n(\%)$ & $64(82.1)$ \\
\hline \multicolumn{2}{|l|}{ Cushing's disease status } \\
\hline De novo Cushing disease, $n(\%)$ & $12(15.4)$ \\
\hline Persistent/recurrent Cushing's disease, $n(\%)$ & $66(84.6)$ \\
\hline Mild hypercortisolism, $n(\%)$ & $14(19.2)$ \\
\hline Moderate hypercortisolism, $n(\%)$ & $34(46.6)$ \\
\hline Severe hypercortisolism, $n(\%)$ & $17(23.3)$ \\
\hline Very severe hypercortisolism, $n(\%)$ & $8(11.0)$ \\
\hline \multicolumn{2}{|l|}{ Physical characteristics, mean (s.D.) } \\
\hline BMI & $30.3(7.1)$ \\
\hline Facial rubora & $1.5(0.9)$ \\
\hline Striaea & $1.0(1.2)$ \\
\hline Bruising $^{b}$ & $0.5(0.8)$ \\
\hline Supraclavicular fat pad ${ }^{b}$ & $1.5(0.9)$ \\
\hline Dorsal fat pad ${ }^{\mathrm{b}}$ & $1.7(0.8)$ \\
\hline \multicolumn{2}{|l|}{ Lab values } \\
\hline mUFC (nmol/24 h), mean (s.D.), median & $\begin{array}{c}782.0(873.6) \\
511\end{array}$ \\
\hline HbA1c (\%), mean (s.D.) & $5.8(0.8)$ \\
\hline Standing diastolic blood pressure $(\mathrm{mmHg})$ & $86.6(12.7)$ \\
\hline Standing systolic blood pressure $(\mathrm{mmHg})$ & $130.0(17.5)$ \\
\hline Sitting diastolic blood pressure $(\mathrm{mmHg})$ & $86.0(11.1)$ \\
\hline Sitting systolic blood pressure $(\mathrm{mmHg})$ & $131.4(15.5)$ \\
\hline \multicolumn{2}{|l|}{ Patient-reported measures, mean (s.D.) } \\
\hline BDI-II & $17.4(10.3)$ \\
\hline CushingQoL & $42.4(21.0)$ \\
\hline Received anti-diabetic treatment during tri & $46(59)$ \\
\hline
\end{tabular}

BDI-II, Beck Depression Inventory II; CushingQOL, Cushing's Quality of Life Questionnaire; mUFC, mean urinary free cortisol.

${ }^{a}$ Study sample consists of patients who completed 12 months of the trial. ${ }^{\mathrm{b}}$ Response categories included none (0), mild (1), moderate (2), or severe (3). 


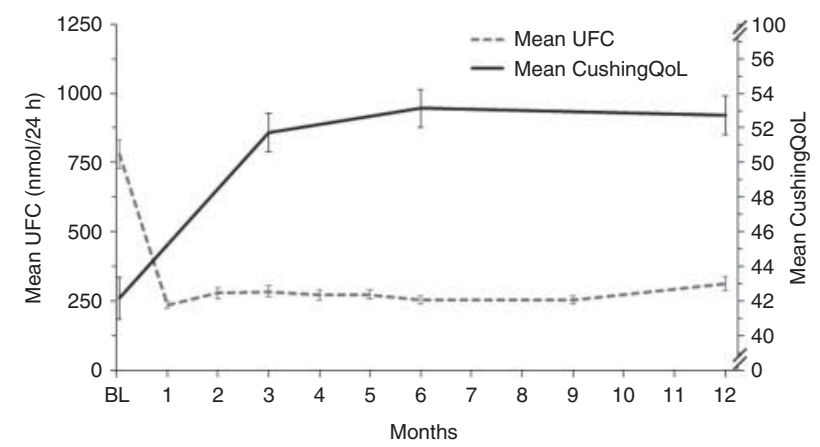

\section{Figure 2}

Changes in mUFC and the CushingQoL among study completers over time. Spearman's rank order correlation $r=-0.40$ with $P<0.01$. CushingQoL, Cushing's Quality of Life Questionnaire; mUFC, mean urinary free cortisol. Error bars represent standard error at the respective months.

both indicating borderline hypertension. The average BMI was $30.3 \mathrm{~kg} / \mathrm{m}^{2}$, which is considered to be overweight or pre-obese (16). The clinical features (facial rubor, striae, bruising, supraclavicular fat pad, and dorsal fat pad) were, on average, mild. The mean BDI-II score of 17 fell in the range of mild depression. Baseline characteristics (i.e. CushingQoL score, UFC, BMI, BDI, age, sex, race, and status of $\mathrm{CD}$ ) were compared between participants who completed the trial and those who did not. The only difference $(P<0.05)$ was that a larger number of males dropped out of the study.

\section{Association between CushingQoL and mUFC}

Figure 2 displays the mean mUFC values and CushingQoL scores at baseline and at all available follow-up visits to month 12. The trend shows that CushingQoL scores increased (indicating improved HRQOL) as mUFC levels declined/improved. The reduction in mUFC and improvement in CushingQoL scores from baseline were sustained throughout the 12-month treatment period. A similar pattern was observed in the full sample analysis including all patients who participated in the trial.

The Spearman's rank order correlation between changes in CushingQoL and changes in mUFC was weak from baseline to month $6(r=-0.05)$. However, a moderate and statistically significant correlation of -0.40 $(P<0.01)$ was observed from baseline to month 12 . The effect sizes for CushingQoL improvements from baseline to month 6 and from baseline to month 12 were moderately large (both effect sizes $=0.53$ ).
Table 2 presents the mean change in CushingQoL from baseline to months 6 and 12, stratified by hypercortisolism level. Patients who achieved a normalized cortisol level or mild hypercortisolism level at month 6 had an average improvement in the CushingQoL of 10-13.5 units from baseline. By month 12 , patients who achieved a normalized cortisol or mild hypercortisolism level had an even greater improvement in the CushingQoL compared with baseline (13-15 units). Patients with moderate hypercortisolism level at month 6 had a 7-unit improvement from baseline in the CushingQoL; by month 12, patients in the moderate level had an average improvement of 3.5 units in the CushingQoL from baseline. Although there was a small sample size $(n=3)$, patients in the severe category at month 6 had an average improvement of 36.8 units in the CushingQoL from baseline, while at month 12 , the average improvement in the CushingQoL from baseline was 7.6 units. By month 6, no patients who completed the trial were in the very severe category. Patients with very severe hypercortisolism at month 12 had an average decrease of 5.2 units in the CushingQoL from baseline.

In the multinomial regression analysis adjusting for covariates, expected CushingQoL score at month 12 was 58.3 for mUFC controlled $(\Delta=11.5$ vs uncontrolled, $P=0.012), 54.5$ for partially controlled $(\Delta=7.7$ vs uncontrolled, $P=0.170$ ), and 46.8 for uncontrolled. The model explained $\sim 40 \%$ of the variation in CushingQoL $\left(r^{2}=0.40\right)$. Based on this regression analysis, only mUFC control status at month 12 and the baseline CushingQoL

Table 2 Mean CushingQoL change score by hypercortisolism level at months 6 and 12. Patients with missing data on CushingQoL change or raw mUFC levels at months 6 and 12 were excluded from the respective analyses.

\begin{tabular}{|c|c|c|c|c|}
\hline \multirow{2}{*}{$\begin{array}{l}\text { Concurrent } \\
\text { hypercortisolism level }^{\mathrm{a}}\end{array}$} & \multicolumn{2}{|c|}{$\begin{array}{l}\text { Change by } \\
\text { month } 6\end{array}$} & \multicolumn{2}{|c|}{$\begin{array}{l}\text { Change by } \\
\text { month } 12\end{array}$} \\
\hline & Mean $^{b}$ & S.D. & Mean $^{b}$ & S.D. \\
\hline $\begin{array}{l}\text { Normal } \\
\qquad(\mathrm{mUFC} \leq 145 \mathrm{nmol} / 24 \mathrm{~h})\end{array}$ & 9.94 & 13.54 & 12.99 & 16.03 \\
\hline $\begin{array}{l}\text { Mild (145 } \mathrm{nmol} / 24 \mathrm{~h} \\
\quad<\mathrm{mUFC} \leq 290 \mathrm{nmol} / 24 \mathrm{~h})\end{array}$ & 13.50 & 13.66 & 14.68 & 21.49 \\
\hline $\begin{array}{l}\text { Moderate }(290 \mathrm{nmol} / 24 \mathrm{~h} \\
<\mathrm{mUFC} \leq 725 \mathrm{nmol} / 24 \mathrm{~h})\end{array}$ & 6.94 & 16.53 & 3.52 & 16.99 \\
\hline $\begin{array}{l}\text { Severe }(725 \mathrm{nmol} / 24 \mathrm{~h} \\
\quad<\mathrm{mUFC} \leq 1450 \mathrm{nmol} / 24 \mathrm{~h})\end{array}$ & 36.81 & 13.39 & 7.64 & 15.36 \\
\hline $\begin{array}{l}\text { Very severe } \\
\qquad(\mathrm{mUFC}>1450 \mathrm{nmol} / 24 \mathrm{~h})\end{array}$ & - & - & -5.21 & 36.83 \\
\hline
\end{tabular}

${ }^{a}$ Concurrent hypercortisolism level refers to hypercortisolism level at month 6 for month 6 change results and hypercortisolism level at month 12 for month 12 change results.

${ }^{\mathrm{b}} P=0.336$ at month 6 and $P=0.067$ at month 12 in trend analysis. 
Table 3 Mean and S.D. of baseline and change score of CushingQoL Item responses by month 12 . Paired $t$-test was conducted to assess the difference in means between baseline and month 12.

\begin{tabular}{|c|c|c|c|}
\hline CushingQoL item & $\begin{array}{l}\text { Baseline, } \\
\text { mean (s.o.) }\end{array}$ & $\begin{array}{l}\text { Change by } \\
\text { month 12, } \\
\text { mean (s.D.) }\end{array}$ & $\begin{array}{l}\text { Change } \\
\text { from base- } \\
\text { line }(\%)\end{array}$ \\
\hline Trouble sleeping & $2.39(1.28)$ & $0.54(1.24)$ & $22.6^{\ddagger}$ \\
\hline $\begin{array}{l}\text { Pain keeping from a } \\
\text { normal life }\end{array}$ & $3.21(1.47)$ & $0.45(1.15)$ & $14.0^{\dagger}$ \\
\hline Delayed wound healing & $3.48(1.35)$ & $0.26(1.31)$ & 7.5 \\
\hline Bruising & $2.49(1.28)$ & $0.23(1.42)$ & 9.2 \\
\hline $\begin{array}{l}\text { Irritable, sudden mood } \\
\text { swings, and angry } \\
\text { outbursts }\end{array}$ & $2.66(1.13)$ & $0.44(1.14)$ & $16.5^{\dagger}$ \\
\hline $\begin{array}{l}\text { Less self-confidence, } \\
\text { insecure }\end{array}$ & $3.04(1.18)$ & $0.45(1.11)$ & $14.8^{\ddagger}$ \\
\hline $\begin{array}{l}\text { Worry about the changes } \\
\text { in physical appearance }\end{array}$ & $1.83(1.11)$ & $0.74(1.14)$ & $40.4^{\ddagger}$ \\
\hline $\begin{array}{l}\text { Less interest for going out, } \\
\text { seeing relatives or friends }\end{array}$ & $2.96(1.24)$ & $0.48(1.44)$ & $16.2^{\dagger}$ \\
\hline $\begin{array}{l}\text { Give up social or leisure } \\
\text { activities }\end{array}$ & $2.97(1.40)$ & $0.59(1.28)$ & $19.9^{\ddagger}$ \\
\hline $\begin{array}{l}\text { Affects everyday activities } \\
\text { (e.g. working and studying) }\end{array}$ & $2.75(1.41)$ & $0.46(1.32)$ & $16.7^{\dagger}$ \\
\hline Difficult to remember things & $2.83(1.21)$ & $0.32(1.23)$ & $11.3 *$ \\
\hline $\begin{array}{l}\text { Worried about health in the } \\
\text { future }\end{array}$ & $1.73(0.98)$ & $0.53(0.98)$ & $30.6^{\ddagger}$ \\
\hline
\end{tabular}

score were significant, independent predictors of the month 12 CushingQoL scores. Age, gender, and hyperglycemia status at month 12 were not significant predictors of CushingQoL scores at 12 months.

\section{Changes in CushingQoL items and total scores over time}

Table 3 presents the extent of improvement on each of the CushingQoL items from baseline to month 12 . On average, ten of the twelve CushingQoL items showed a significant improvement by month 12 , with the biggest improvements observed on the items 'worried about changes in physical appearance', 'worried about health in the future', 'trouble sleeping', and 'give up social/leisure activities' (all $\geq 20 \%$ improvement from baseline).

Figure 3 shows the relationship between mUFC control status and the percentage of patients meeting the CushingQoL MID threshold. Based on the 10.1 MID threshold and stratifying by mUFC control status at the primary endpoint at month 6 , the percentages of patients classified as being CushingQoL responders at month 12 were $63.0 \%$ (17 out of 27 ) in the controlled group, $58.8 \%$ (ten out of 17 ) in the partially controlled group, and $37.9 \%$ (11 out of 29) in the uncontrolled group. A similar pattern was observed when stratifying by month 12 mUFC control status, where percentages of CushingQoL responders at month 12 were $56.7 \%$ (17 out 30 ) in the controlled group, $69.2 \%$ (nine out 13 ) in the partially controlled group, and $33.3 \%$ (nine out of 27) in the uncontrolled group. The percentage of patients whose mUFC levels were controlled at month 6 and maintained at month 12, and who also met the CushingQoL responder threshold, were 63.2\% (12 out of 19), whereas, notably, the percentage of patients who were partially controlled at both months 6 and 12 or who had controlled mUFC at one time point and partially controlled mUFC at another time point and met the CushingQoL responder threshold at month 12 was higher at $73.3 \%$ (11 out of 15$)$. The percentage of patients who

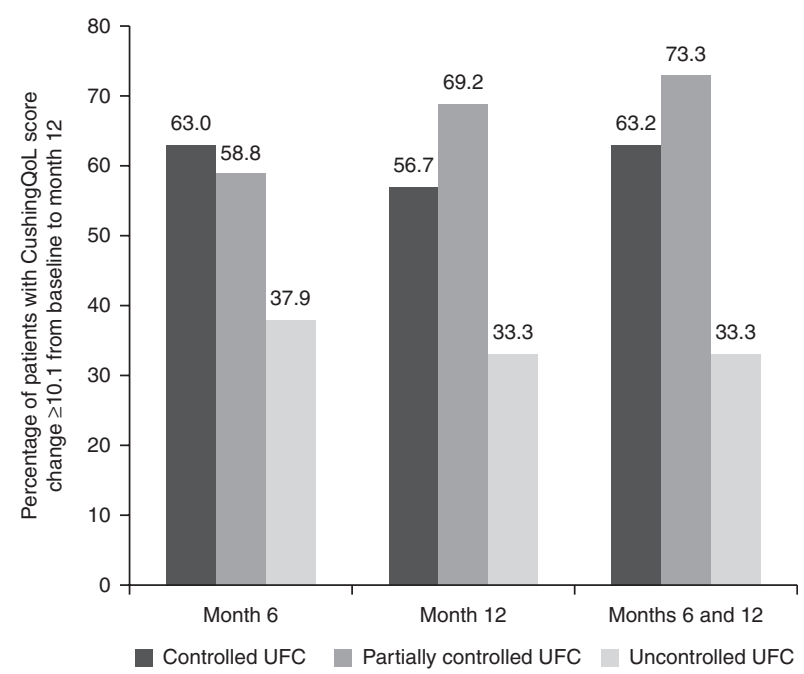

\section{Figure 3}

Percentage of patients with CushingQoL improvement $\geq 10.1$ from baseline to month 12 by mUFC control status at months 6 and 12. Missing data were not imputed. Patients were excluded from this analysis if they did not have a CushingQoL change score and/or UFC scores. UFC status was defined as controlled at months 6 and 12 if patients were controlled at both time points. Patients were considered partially controlled at months 6 and 12 if they were partially controlled at both time points, OR if they were controlled at one time point and partially controlled at the other time point. Uncontrolled UFC status included patients who had an uncontrolled UFC status at both time points. The numbers of patients in the controlled, partially controlled, and uncontrolled groups were: 27,17 , and 29 respectively at month $6 ; 30,13$, and 27 respectively at month 12 ; and 19,15 , and 18 respectively at months 6 and 12 . 
had uncontrolled UFC at both months 6 and 12 and met the CushingQoL responder threshold at months 6 and 12 were markedly lower at $33.3 \%$ (six out of 18 ). Also, the median percentage improvement in CushingQoL scores was $\sim 30 \%$ in the controlled group and only $9.8 \%$ in the uncontrolled group.

\section{Association between HRQOL and other CD symptoms and signs}

To assess the relationship between HRQOL and depression among CD patients, the correlations between baseline BDI-II and baseline CushingQoL item and total scores were estimated. The Spearman's rank sum correlation coefficients ranged between -0.27 and -0.70 (all $P<0.001$; Table 4). The strongest correlation was observed between the CushingQoL total score and the BDI-II total score $(r=-0.70)$, indicating that greater impairment in CD-related HRQOL is associated with greater depression severity. Among the CushingQoL items, the strongest correlation with depression was observed with the item on 'less self-confidence, insecure', followed by 'affects everyday activities (e.g. working/studying)', 'give up social/leisure activities', 'pain keeps from a normal life', 'irritable, sudden mood swings, and angry outbursts', and 'difficult to remember things' (all $r \geq 0.50$ ).

Significant correlations $(P<0.01)$ were also observed between changes in CushingQoL scores and changes in BMI ( $r=-0.39$ at 6 months and -0.31 at 12 months), weight ( $r=-0.41$ at 6 months and -0.32 at 12 months), and BDI-II score $(r=-0.54$ at 6 months and -0.59

Table 4 Correlations between Beck Depression Inventory II (BDI-II) and CushingQoL item and total scores at baseline.

CushingQoL
Trouble sleeping
Pain keeping from a normal life
Delayed wound healing
Bruising
Irritable, sudden mood swings, and angry
outbursts
Less self-confidence, insecure
Worry about the changes in physical
appearance
Less interest for going out, seeing relatives
or friends
Give up social or leisure activities
Affects everyday activities (working and
studying)
Difficult to remember things
Worried about health in the future
CushingQoL score

BDI-II P value

$-0.47<0.001$

$-0.52<0.001$

$-0.31 \quad 0.007$

$-0.27 \quad 0.020$

$-0.51<0.001$

$-0.59<0.001$

$-0.31 \quad 0.006$

$-0.45<0.001$

$-0.55<0.001$

$-0.57<0.001$

$-0.50<0.001$

$-0.48<0.001$

$-0.70<0.001$ at 12 months). A significant 4.9 reduction in the BDI-II score was observed at 12 months in UFC responders. No correlation was observed between changes in CushingQoL and changes in waist circumference, blood pressure, facial rubor, striae, bruising, supraclavicular fat pad, and dorsal fat pad.

\section{Discussion}

This study investigated the treatment effect of pasireotide on HRQOL using data from a phase III clinical trial of patients with $\mathrm{CD}$, and assessed relationships between HRQOL and UFC control and other signs and symptoms of CD. A substantial HRQOL burden in patients with CD has been documented previously using the generic SF-36 Health Survey $(8,17,18,19)$. Utilizing the disease-specific measure, CushingQoL, to capture the HRQOL concerns that are important to patients with $\mathrm{CD}$, is necessary to fully understand the benefits of treatment from the perspective of a patient with CD.

Pasireotide can significantly improve HRQOL in CD patients as measured by the CushingQoL. The extent of the improvement on CushingQoL moderately correlated with improvement in mUFC across the 12-month treatment period. The majority of patients whose mUFC levels were controlled or partially controlled at month 6 , and who maintained at least partially controlled status at month 12, had a clinically meaningful improvement on CushingQoL. At month 12 , over $60 \%$ of patients with controlled mUFC levels and over $70 \%$ of patients with controlled or partially controlled mUFC levels reached the CushingQoL MID for improvement, whereas only onethird of patients in the uncontrolled group reached this threshold. It was also observed that by month 12 , patients with a normalized cortisol level or mild hypercortisolism had substantial improvement in their CushingQoL scores. The mean CushingQoL improvement for patients in these two categories reached the MID threshold. Patients who maintained moderate or severe hypercortisolism level experienced little improvement in CushingQoL scores. The evidence herein suggests that declining UFC levels are associated with improvements in HRQOL.

This relationship between CushingQoL score and mUFC status was further supported by the multivariable regression analysis results. The only factors significantly associated with month 12 CushingQoL score were the baseline CushingQoL score and month 12 mUFC control status. After adjusting for other factors, patients with controlled mUFC at month 12 had a significantly better CushingQoL score than the uncontrolled patients. 
Age, gender, hyperglycemia, and partially controlled mUFC at month 12 were not significantly associated with the CushingQoL scores at month 12 . A sensitivity analysis found no significant association between month 12 CushingQoL score and different doses of pasireotide (i.e. 600 and $900 \mu \mathrm{g}$ ).

The improvements in HRQOL after pasireotide treatment were captured in the responses to multiple CushingQoL items. Many of such items are measured in other HRQOL instruments (e.g. sleep adequacy, pain, psychological distress, role and social activities, cognitive functioning, and worry about future health). Others are known to be important to patients with $\mathrm{CD}$ specifically (e.g. 'worry about the changes in physical appearance'). At the end of the pasireotide trial (month 12), except for two items (i.e. wounds and bruising), the changes in all other CushingQoL items indicated significant relative improvements from baseline, ranging from 11.3 to $40.4 \%$. The biggest improvement was evident in the item 'worry about the changes in physical appearance'.

Changes in CushingQoL were moderately correlated with changes in clinical signs and symptoms assessed by BMI, weight, and depression. Changes in CushingQoL were not correlated with changes in blood pressure and clinical features such as facial rubor, striae, bruising, and posterior fat pad. This was probably due to the fact that the majority of patients had absent or mild CD features, leaving little room for improvement. Although it is well established that symptoms and HRQOL are associated, it is not clear whether clinical features would have an impact on patients' QOL.

This study builds on previous work on HRQOL in patients with $\mathrm{CD}$ and $\mathrm{CS}(2,5,8,17,20,21,22,23)$. Most of the previously published studies have been retrospective or have used small sample sizes. Several studies used single-item questions to assess the impact of CD or CS on HRQOL, or used generic instruments such as the SF-36 Health Survey (24). For example, van Aken et al. (2) evaluated HRQOL in a retrospective study of 58 patients who had undergone treatment for $\mathrm{CD}$ and compared scores on four generic HRQOL instruments, including the SF-36, with published age-adjusted norms. Patients with CD had worse HRQOL scores than the general population, specifically in the domains of fatigue and physical ability. Lindsay et al. (8) conducted a study including: i) comparison of SF-36 domain scores in 23 patients before and after transsphenoidal surgery and ii) a retrospective study conducted comparing 343 CS patients in long-term remission who had undergone surgeries over a span of 25 years to age- and sex-matched controls.
Post-operative SF-36 domain scores of the 23 patients improved over pretreatment values. The average scores of six SF-36 domains of these CD patients did not differ significantly from those of the age- and sex-matched USA population. However, their physical component scores (i.e. physical functioning, role limitation due to physical health, and physical component summary) remained significantly impaired. Over the long term, the differences between 343 post-surgery CS patients in remission and their age- and sex-matched controls were large in all SF-36 domains, except for moderate impairment in mental health and bodily pain domains. Lindsay et al. concluded that CS was associated with impairment in HRQOL, which only partially resolves after surgery.

This study extends previous research, providing evidence that patients with CD have impaired HRQOL, using a disease-specific HRQOL tool specifically designed for patients with CD. By using CushingQoL, this study focused the HRQOL domains of greatest concern for patients with CD. As reflected in current analyses, successful treatment could help these patients achieve clinically meaningful HRQOL improvement, particularly among those who achieved biochemical control.

The longitudinal design of the phase III clinical trial was a major strength for examining the HRQOL improvements following treatment; however, there were several limitations of the study. One main limitation was the lack of a control group. At the time of this trial there were no approved medical therapies for $\mathrm{CD}$ and, for ethical considerations, all patients received active treatment. This study was also designed to allow open label dose up-titration starting at month 3 if their UFC was $>2 \times$ ULN or above the baseline UFC. While this could potentially bias the patient-reported HRQOL outcome, it would not affect the determination of the mUFC control status at the primary study endpoint at month 6 . This is because per protocol, patients who had dose up-titration before month 6 were automatically assigned the mUFC uncontrolled status at month 6 . Another limitation was a lack of data utilizing the generic HRQOL instrument in this study, leaving a question about association between diseasespecific HRQOL and broader general HRQOL in this population, as demonstrated in clinical practice (11). A further limitation stemmed from the definition of MID by Nelson et al. (13), which used the same population as the current study. In addition, we did not assess whether side effects could be dose dependent and whether such a potential effect could have an impact on the HRQOL benefit. This is an interesting topic that warrants future research. Finally, an additional concern was the small 
sample size, with 78 patients completing the 12-month trial. In a sensitivity analysis of the full study sample, improvements in CushingQoL and mUFC over time were consistent with those observed in the 12-month completers. Future research should collect long-term data on UFC and both CD-specific and generic HRQOL to further investigate if long-term biochemical control leads to sustained improvements in HRQOL.

Evidence from this study supports the associations between HRQOL and biochemical control status, other symptoms/signs and mental health (e.g. as measured by BDI-II) in patients with CD. This study found that pasireotide can be effective in improving HRQOL in patients with $\mathrm{CD}$. Improvements were greatest when patients achieved a controlled UFC status (the CD biochemical marker) upon treatment. The disease-specific measure, CushingQoL, adequately captured the HRQOL burden of CD at baseline and identified improvements in HRQOL that were meaningful to patients upon effective treatment.

\section{Declaration of interest}

A Forsythe and M Maldonado were previously employed by Novartis (USA). J E Ware was a consultant on this project and is an employee of University of Massachusetts Medical School. M Yang and J E Signorovitch were consultants on this project and are employees of Analysis Group, Inc. $\mathrm{L}$ McLeod and L M Nelson were consultants on this project, and are employees of RTI-HS. S M Webb was a consultant on this project and is an employee at Universitat Autònoma de Barcelona; she has received lecture fees from Novartis. $X$ Badia was a consultant on this project and was an employee of IMS health. M Gadelha has received lecture fees from Novartis, Ipsen, and Pfizer as well as research grants from Novartis and Pfizer. M Gadelha also serves on an advisory board for Novartis. W Zgliczynski has received lecture fees and research grants from Novartis and Ipsen. $C$ de Block and L Portocarrero-Ortiz have no conflict of interest.

\section{Funding}

This work was supported by Novartis Pharmaceuticals Corporation.

\section{Acknowledgements}

This work was a collaboration between Novartis, IMS Health, RTI-HS, and Analysis Group, Inc. with financial support from Novartis. The authors thank Yanqiong Zhang and Sonia Pulgar (former Novartis employees), Eric Q Wu (Analysis Group, Inc.), and Theresa Coles (RTI-HS) for their contributions to the interpretation of data. The authors thank the sites and patients included in the clinical study.

\section{References}

1 Colao A, Petersenn S, Newell-Price J, Findling JW, Gu F, Maldonado M, Schoenherr U, Mills D, Salgado LR \& Biller BM. A 12-month phase 3 study of pasireotide in Cushing's disease. New England Journal of Medicine 2012366 914-924. (doi:10.1056/NEJMoa1105743)

2 Van Aken MO, Pereira AM, Biermasz NR, Van Thiel SW, Hoftijzer HC, Smit JW, Roelfsema F, Lamberts SW \& Romijn JA. Quality of life in patients after long-term biochemical cure of Cushing's disease. Journal of Clinical Endocrinology and Metabolism 200590 3279-3286. (doi:10.1210/jc.2004-1375)

3 Gotch PM. Cushing's syndrome from the patient's perspective. Endocrinology and Metabolism Clinics of North America 199423 607-617.

4 Sippel RS, Elaraj DM, Kebebew E, Lindsay S, Tyrrell JB \& Duh Q-Y. Waiting for change: symptom resolution after adrenalectomy for Cushing's syndrome. Surgery 2008144 1054-1061. (doi:10.1016/ j.surg.2008.08.024)

5 Sonino N \& Fava GA. Psychosomatic aspects of Cushing's disease. Psychotherapy and Psychosomatics 199867 140-146. (doi:10.1159/ 000012274)

6 Webb SM, Badia X, Barahona MJ, Colao A, Strasburger CJ, Tabarin A, Van Aken MO, Pivonello R, Stalla G, Lamberts SW et al. Evaluation of health-related quality of life in patients with Cushing's syndrome with a new questionnaire. European Journal of Endocrinology $2008 \mathbf{1 5 8}$ 623-630. (doi:10.1530/EJE-07-0762)

7 Arnaldi G, Angeli A, Atkinson AB, Bertagna X, Cavagnini F, Chrousos GP, Fava GA, Findling JW, Gaillard RC, Grossman AB et al. Diagnosis and complications of Cushing's syndrome: a consensus statement. Journal of Clinical Endocrinology and Metabolism $2003 \mathbf{8 8}$ 5593-5602. (doi:10.1210/jc.2003-030871)

8 Lindsay JR, Nansel T, Baid S, Gumowski J \& Nieman LK. Long-term impaired quality of life in Cushing's syndrome despite initial improvement after surgical remission. Journal of Clinical Endocrinology and Metabolism 200691 447-453. (doi:10.1210/jc.2005-1058)

9 Thompson SK, Hayman AV, Ludlam WH, Deveney CW, Loriaux DL \& Sheppard BC. Improved quality of life after bilateral laparoscopic adrenalectomy for Cushing's disease: a 10-year experience. Annals of Surgery 2007245 790-794. (doi:10.1097/01.sla.0000251578.03883.2f)

10 Boscaro M, Ludlam WH, Atkinson B, Glusman JE, Petersenn S, Reincke M, Snyder P, Tabarin A, Biller BM, Findling J et al. Treatment of pituitary-dependent Cushing's disease with the multireceptor ligand somatostatin analog pasireotide (SOM230): a multicenter, phase II trial. Journal of Clinical Endocrinology and Metabolism 200994 115-122. (doi:10.1210/jc.2008-1008)

11 Santos A, Resmini E, Martínez-Momblán MA, Crespo I, Valassi E, Roset M, Badia X \& Webb SM. Psychometric performance of the CushingQoL questionnaire in conditions of real clinical practice. European Journal of Endocrinology 2012167 337-342. (doi:10.1530/ EJE-12-0325)

12 Norman GR, Sloan JA \& Wyrwich KW. Interpretation of changes in health-related quality of life: the remarkable universality of half a standard deviation. Medical Care 200341 582-592.

13 Nelson LM, Forsythe A, McLeod L, Pulgar S, Maldonado M, Coles T, Zhang Y, Webb SM \& Badia X. Psychometric evaluation of the Cushing's Quality-of-Life Questionnaire. Patient 20136 113-124. (doi:10.1007/s40271-013-0012-5)

14 Beck AT, Steer RA \& Brown GK. Manual for the Beck Depression Inventory-II. San Antonio, TX: Psychological Corporation, 1996.

15 Cohen J. The Analysis of Variance. In Statistical Power Analysis for the Behavioral Sciences, 2nd edn. Hillsdale, NJ: Lawrence Erlbaum Associates, 1988.

16 WHO Expert Committee. Physical status: the use and interpretation of anthropometry. In Technical Report, Series No. 854. Geneva: World Health Organization (available at: http://www.who.int/childgrowth/ publications/physical_status/en/index.html, accessed May 19, 2013), 1995.

17 Hawn MT, Cook D, Deveney C \& Sheppard BC. Quality of life after laparoscopic bilateral adrenalectomy for Cushing's disease. Surgery 2002132 1064-1068. (doi:10.1067/msy.2002.128482) 
18 Johnson MD, Woodburn CJ \& Vance ML. Quality of life in patients with a pituitary adenoma. Pituitary 20036 81-87. (doi:10.1023/B:PITU. 0000004798.27230.ed)

19 Lindholm J, Juul S, Jorgensen JO, Astrup J, Bjerre P, Feldt-Rasmussen U, Hagen C, Jorgensen J, Kosteljanetz M, Kristensen L et al.

Incidence and late prognosis of Cushing's syndrome: a populationbased study. Journal of Clinical Endocrinology and Metabolism 200186 117-123.

20 Dorn LD, Burgess ES, Friedman TC, Dubbert B, Gold PW \& Chrousos GP. The longitudinal course of psychopathology in Cushing's syndrome after correction of hypercortisolism. Journal of Clinical Endocrinology and Metabolism 199782 912-919.
21 Pikkarainen L, Sane T \& Reunanen A. The survival and well-being of patients treated for Cushing's syndrome. Journal of Internal Medicine 1999245 463-468. (doi:10.1046/j.1365-2796.1999.00483.x)

22 Forget H, Lacroix A \& Cohen H. Persistent cognitive impairment following surgical treatment of Cushing's syndrome. Psychoneuroendocrinology 20022 367-383. (doi:10.1016/S0306-4530(01)00059-2)

23 Heald AH, Ghosh S, Bray S, Gibson C, Anderson SG, Buckler H \& Fowler HL. Long-term negative impact on quality of life in patients with successfully treated Cushing's disease. Clinical Endocrinology 2004 61 458-465. (doi:10.1111/j.1365-2265.2004.02118.x)

24 Ware JKM \& Dewey JE. How to Score Version Two of the SF-36 ${ }^{\circledR}$ Health Survey (standard and acute forms), 3rd edn. Lincoln, RI: QualityMetric, Inc., 2000.

Received 10 December 2013

Revised version received 13 April 2014

Accepted 22 April 2014 